DESY $97-143$

hep-ph/9707488

WUE-ITP-97-22

July 1997

(Revised 10/97)

\title{
On the Resummed Gluon Anomalous Dimension and Structure Functions at Small $\boldsymbol{x}$
}

\author{
Johannes Blümlein ${ }^{a}$ and Andreas Vogt ${ }^{b}$ \\ ${ }^{a}$ DESY-Zeuthen, Platanenallee 6, D-15735 Zeuthen, Germany \\ ${ }^{b}$ Institut für Theoretische Physik, Universität Würzburg \\ Am Hubland, D-97074 Würzburg, Germany
}

\begin{abstract}
The impact of the recently evaluated 'irreducible' contributions to the resummed next-to-leading logarithmic small- $x$ anomalous dimension $\gamma_{g g}$ is evaluated for the unpolarized parton densities and structure functions of the nucleon. These new terms diminish the gluon distribution and are found to overcompensate the enhancement caused by the resummed leading logarithmic small- $x$ anomalous dimension and the quarkonic contributions beyond next-to-leading order.
\end{abstract}

PACS: 12.38.Cy, 13.60.Hb

Phys. Rev. D57 (1998) 1 
The structure functions of the proton in unpolarized deep-inelastic scattering (DIS) show a steep rise towards very small Bjorken- $x$ values, which becomes stronger with increasing resolution $Q^{2}$. This behavior is a consequence both of the shape of the non-perturbative quark singlet and gluon initial distributions $-x \Sigma$ and $x g$ rise roughly like $x^{-0.2}$ for a starting scale $Q_{0}^{2} \simeq 4 \mathrm{GeV}^{2}$ - and of the form of the evolution kernels governing the renormalization group equations of the mass singularities. The anomalous dimensions $\gamma_{i j}$ for the evolution of the parton densities, as well as the Wilson coefficients $C_{n}$ of the structure functions, contain large logarithmic small- $x$ contributions. In order to arrive at a reliable theoretical framework at very small $x$, the resummation of these terms may be necessary to all orders in the strong coupling $\alpha_{s}\left(Q^{2}\right)$. For the unpolarized singlet case considered here the dominant contributions take, in Mellin- $N$ space, the form $\alpha_{s}^{l+k} /(N-1)^{k}, l \geq 0$.

The resummation of $\gamma_{g g}$ and $\gamma_{g q}$ in the leading small- $x$ approximation $(\mathrm{L} x), l=0$, was performed long ago [1]. $\gamma_{g g}^{(0)}(N)$ is obtained as the solution of

$$
1=\frac{\bar{\alpha}_{s}}{N-1} \chi_{0}\left(\gamma_{g g}^{(0)}\right)
$$

with $\bar{\alpha}_{s}=C_{A} \alpha_{s}\left(Q^{2}\right) / \pi, C_{A}=N_{c}=3, C_{F}=4 / 3$ and

$$
\chi_{0}(\gamma)=2 \psi(1)-\psi(\gamma)-\psi(1-\gamma),
$$

furthermore $\gamma_{g q}^{(0)}=\left(C_{F} / C_{A}\right) \gamma_{g g}^{(0)}$. The quark anomalous dimensions $\gamma_{q q}$ and $\gamma_{q g}$, on the other hand, receive contributions for $l \geq 1$ only. The $l=1$ terms were derived in ref. [2], together with the corresponding resummations for the coefficient functions $C_{2}$ and $C_{L}$. The large effects of these quantities on the small- $x$ behavior of the DIS structure functions were subsequently studied in detail [3-7]. In those investigations the small- $x$ resummation of the gluon anomalous dimension $\gamma_{g g}$ to next-to-leading order small- $x$ (NL $x$ ) accuracy could not be taken into account. This resummation has now been performed for the quarkonic contributions [8-11] proportional to the number of quark flavors $N_{f}$. Recently also the 'irreducible' gluonic terms $\propto C_{A}$ have been derived [12], i.e., those contributions which are energy-scale independent in the framework of ref. [10] underlying that calculation. The corresponding terms of $\gamma_{g q}$, however, still remain to be determined.

In this note we investigate the impact of these new resummed contributions to $\gamma_{g g}$ on the evolution of the parton densities and the proton structure functions $F_{2}\left(x, Q^{2}\right)$ and $F_{L}\left(x, Q^{2}\right)$, for the first time including calculated subleading terms into the renormalization group analysis. Hence the comparison of the results to the findings of previous studies [3-7] should allow for improved estimates of the convergence of the small- $x$ resummation approximation, despite a fully quantitative, scheme-independent NL $x$ analysis not being possible at present.

As will be demonstrated below, the effect of the new contributions to the resummed anomalous dimension $\gamma_{g g}$ is very large and opposite to that of the previously known resummed terms. This implies, already at the present stage, considerable changes particularly for gluon-dominated quantities, which partly modify conclusions obtained in previous numerical investigations [3-6]. A detailed account of the solution of the evolution equations in the presence of all-order anomalous dimensions and coefficient functions will be given in a forthcoming publication [13].

As shown in ref. [9] the larger eigenvalue of the singlet anomalous dimension matrix, $\gamma_{+}(N)$, may be obtained in the $Q_{0}$ scheme [14] as the solution of

$$
1=\frac{\bar{\alpha}_{s}}{N-1}\left[\chi_{0}\left(\gamma_{+}\right)+\alpha_{s} \chi_{1}\left(\gamma_{+}\right)\right],
$$


where the second term is the sum of [9, 12]

$$
\begin{aligned}
\alpha_{s} \chi_{1}^{q \bar{q}}=\frac{N_{f} \alpha_{s}}{6 \pi} & {\left[\frac{1}{2}\left(\chi_{0}^{2}(\gamma)+\chi_{0}^{\prime}(\gamma)\right)-\frac{5}{3} \chi_{0}-\frac{1}{2 N_{c}^{2}}\left(\frac{\pi}{\sin (\pi \gamma)}\right)^{2} \frac{3 \cos (\pi \gamma)}{1-2 \gamma} \frac{2+3 \gamma(1-\gamma)}{(1+2 \gamma)(3-2 \gamma)}\right] } \\
\alpha_{s} \chi_{1}^{g g}=\frac{C_{A} \alpha_{s}}{4 \pi}[ & {\left[\frac{11}{6}\left(\chi_{0}^{2}(\gamma)+\chi_{0}^{\prime}(\gamma)\right)+\left(\frac{67}{9}-\frac{\pi^{2}}{3}\right) \chi_{0}+\left(6 \zeta(3)+\frac{\pi^{2}}{3 \gamma(1-\gamma)}+\tilde{h}(\gamma)\right)\right.} \\
& \left.-\left(\frac{\pi}{\sin (\pi \gamma)}\right)^{2} \frac{\cos (\pi \gamma)}{3(1-2 \gamma)}\left(11+\frac{\gamma(1-\gamma)}{(1+2 \gamma)(3-2 \gamma)}\right)\right]
\end{aligned}
$$

The function $\tilde{h}(\gamma)$ in eq. (5) is given by

$$
\tilde{h}(\gamma) \simeq \sum_{k=1}^{3} a_{k}\left(\frac{1}{k+\gamma}+\frac{1}{1+k-\gamma}\right)
$$

with $a_{1}=0.72, a_{2}=0.28$ and $a_{3}=0.16$ [12]. From these results the irreducible NL $x$-contribution to $\gamma_{g g}$ is then inferred by [15]

$$
\gamma_{g g}^{(1)}-\frac{\beta_{0}}{4 \pi} \alpha_{s}^{2} \frac{d}{d \alpha_{s}} \ln \left(\gamma_{g g}^{(0)} \sqrt{-\chi_{0}^{\prime}\left(\gamma_{g g}^{(0)}\right)}\right)=\gamma^{+(1)}-\frac{C_{F}}{C_{A}} \gamma_{q g}^{(1)} \equiv-\frac{\alpha_{s} \chi_{1}\left(\gamma_{g g}^{(0)}\right)}{\chi_{0}^{\prime}\left(\gamma_{g g}^{(0)}\right)}-\frac{C_{F}}{C_{A}} \gamma_{q g}^{(1)} .
$$

Our subsequent numerical analysis will be performed in the DIS factorization scheme. Here $\gamma_{g g}^{(1)}$ is represented as

$$
\begin{aligned}
\gamma_{g g, \mathrm{DIS}}^{(1)} & =\gamma_{g g, Q_{0}}^{(1)}+\frac{\beta_{0}}{4 \pi} \alpha_{s}^{2} \frac{d \ln R\left(\alpha_{s}\right)}{d \alpha_{s}}+\frac{C_{F}}{C_{A}}\left[1-R\left(\alpha_{s}\right)\right] \gamma_{q g, Q_{0}}^{(1)} \\
& =\alpha_{s} \sum_{k=1}^{\infty}\left[\frac{N_{f}}{6 \pi}\left(d_{g g, k}^{q \bar{q},(a)}+\frac{C_{F}}{C_{A}} d_{g g, k}^{q \bar{q},(b)}\right)+\frac{C_{A}}{6 \pi} d_{g g, k}^{g g}+\frac{\beta_{0}}{4 \pi} \hat{r}_{k}\right]\left(\frac{\bar{\alpha}_{s}}{N-1}\right)^{k-1} \\
& \equiv \bar{\alpha}_{s} \sum_{k=0}^{\infty} b_{k}^{g,(1)}\left(\frac{\bar{\alpha}_{s}}{N-1}\right)^{k-1}
\end{aligned}
$$

with $R\left(\alpha_{s}\right)$ defined in ref. [2] and $\beta_{0}=(11 / 3) C_{A}-(2 / 3) N_{f}$. Tables of the expansion coefficients $d_{g g, k}^{q \bar{q},(a, b)}, d_{g g, k}^{g g}$ and $\hat{r}_{k}$ may be found in ref. [13]. Here we list for brevity only the numerical values of the first 15 coefficients $b_{k}^{g,(0)}$ and $b_{k}^{g,(1)}$ for the L $x$ and NL $x$ series for $N_{f}=4$, see Table 1 . Note that the new terms $b_{0,1}^{g,(1)}$ agree with the corresponding results from fixed-order perturbation theory already taking into account the 'irreducible' part of $\gamma_{g g}^{(1)}$ only. Collecting all presently available information, the anomalous dimensions for the resummed unpolarized singlet evolution in the DIS scheme are given by

$$
\begin{aligned}
\gamma\left(N, \bar{\alpha}_{s}\right)_{\mathrm{DIS}}= & \bar{\alpha}_{s} \gamma_{0}(N)+\bar{\alpha}_{s}^{2} \gamma_{1}(N)_{\mathrm{DIS}} \\
& +\sum_{k=2}^{\infty}\left(\frac{\bar{\alpha}_{s}}{N-1}\right)^{k+1}\left[\left(\begin{array}{cc}
0 & 0 \\
C_{F} / C_{A} & 1
\end{array}\right) b_{k}^{g,(0)}+(N-1)\left(\begin{array}{cc}
C_{F} / C_{A} b_{k}^{q,(1)} & b_{k}^{q,(1)} \\
0 & b_{k}^{g,(1)}
\end{array}\right)\right] .
\end{aligned}
$$

Here $\gamma_{0}$ and $\gamma_{1}$ denote the leading and next-to-leading order singlet anomalous dimension matrices.

The resummed terms beyond $O\left(\alpha_{s}^{2}\right)$ in eq. (10) do not comply with the energy-momentum sum rule for the parton densities, which requires

$$
\gamma_{q q}\left(N, \alpha_{s}\right)+\gamma_{g q}\left(N, \alpha_{s}\right)=0, \quad \gamma_{q g}\left(N, \alpha_{s}\right)+\gamma_{g g}\left(N, \alpha_{s}\right)=0
$$




\begin{tabular}{||r||c|c|c||}
\hline \hline$k$ & $b_{k}^{g,(0)}$ & $b_{k}^{g,(1)}$ & $b_{k}^{g,(1)} / b_{k}^{g,(0)}$ \\
\hline \hline 0 & $1.000 \mathrm{E}+00$ & $-1.139 \mathrm{E}+00$ & -1.14 \\
1 & $0.000 \mathrm{E}+00$ & $-8.519 \mathrm{E}-01$ & \\
2 & $0.000 \mathrm{E}+00$ & $3.167 \mathrm{E}-01$ & \\
3 & $2.404 \mathrm{E}+00$ & $-1.166 \mathrm{E}+01$ & -4.85 \\
4 & $0.000 \mathrm{E}+00$ & $-9.104 \mathrm{E}+00$ & \\
5 & $2.074 \mathrm{E}+00$ & $-1.554 \mathrm{E}+01$ & -7.49 \\
6 & $1.734 \mathrm{E}+01$ & $-1.511 \mathrm{E}+02$ & -8.71 \\
7 & $2.017 \mathrm{E}+00$ & $-1.350 \mathrm{E}+02$ & -66.95 \\
8 & $3.989 \mathrm{E}+01$ & $-4.513 \mathrm{E}+02$ & -11.31 \\
9 & $1.687 \mathrm{E}+02$ & $-2.226 \mathrm{E}+03$ & -13.19 \\
10 & $6.999 \mathrm{E}+01$ & $-2.533 \mathrm{E}+03$ & -36.19 \\
11 & $6.613 \mathrm{E}+02$ & $-1.006 \mathrm{E}+04$ & -15.21 \\
12 & $1.945 \mathrm{E}+03$ & $-3.540 \mathrm{E}+04$ & -18.20 \\
13 & $1.718 \mathrm{E}+03$ & $-5.245 \mathrm{E}+04$ & -30.54 \\
14 & $1.064 \mathrm{E}+04$ & $-2.060 \mathrm{E}+05$ & -19.35 \\
\hline \hline
\end{tabular}

Table 1: The expansion coefficients $b_{k}^{g,(0)}$ and $b_{k}^{g,(1)}$ for the small- $x$ resummed anomalous dimension $\gamma_{g g}$. The latter quantities are given for four active flavors. For comparison to previously employed estimates [5] also the ratios of these coefficients are shown.

This relation is satisfied by the fixed-order anomalous dimensions order by order in $\alpha_{s}$. The method to restore the sum rule with the least impact on the small- $x$ results is a (diagonal) subtraction at $N=2$, i.e., the addition of appropriate $\delta(1-x)$ contributions to the higherorder quark-quark and gluon-gluon splitting functions. We will label this prescription as $(A)$ below. Other possibilities are the inclusion of somewhat less singular $1 /(N-1)$ terms, later to be superseded by explicit calculations. In this manner a rough estimate can be obtained of the possible effect of subleading small- $x$ contributions to the higher-order anomalous dimensions, particularly in those cases where only the first term is known currently. We will illustrate this procedure by the prescription $(D)$ given by

$$
\gamma_{i j}(N) \rightarrow \gamma_{i j}(N)\left(1-2[N-1]+[N-1]^{3}\right) \text { for } i j=q q, q g, g q
$$

The terms $\propto[N-1]$ in $\gamma_{g g}$ are taken from eq. (9), hence only the terms $\propto[N-1]^{3}$ in this quantity are adjusted according to eq. (11).

We are now ready to discuss the numerical effects of the small- $x$ resummations. For definiteness, we choose the initial distributions of the $\operatorname{MRS}\left(\mathrm{A}^{\prime}\right)$ global fit 16 . Both the gluon and the sea quark densities behave as $x^{-0.17}$ for $x \rightarrow 0$ at the starting scale of $Q_{0}^{2}=4 \mathrm{GeV}^{2}$. The evolution is performed for four massless flavors, and also $\Lambda^{(4)}=231 \mathrm{MeV}$ is adopted from the MRS analysis. We stress that these results are mainly theoretical illustrations. Detailed data analyses would require some flexibility of the input gluon density at small $x$, which is only indirectly constrained by structure function data, as well as the inclusion of heavy-flavor mass effects.

Figure 1 displays the evolution of the proton singlet quark and gluon (momentum) distributions, $x \Sigma\left(x, Q^{2}\right)$ and $x g\left(x, Q^{2}\right)$, in the DIS scheme. Different resummation approximations are compared with the NLO results (full lines). The results with the new $b_{k}^{g,(1)}$ terms omitted are marked by NL $x_{q}$. 
In the quark sector the resummation corrections using prescription $(A)$ are very large, e.g., they exceed a factor of four at $x=10^{-5}$ and $Q^{2}=100 \mathrm{GeV}^{2}$. This huge correction is entirely dominated by the quarkonic (upper row) anomalous dimensions. Omitting the $b_{k}^{g,(1)}$ contributions, and even ignoring the L $x$ terms (that case is not shown in the figure), has an impact of less than about $20 \%$ on the singlet distribution [3, 5]. The quark evolution is, however, very much affected by possible subleading contributions to $\gamma_{q g}$ and $\gamma_{q q}$, even by such terms which are smaller than those now found for $\gamma_{g g}$. This is illustrated by prescription $(D)$, where the less singular pieces actually overcompensate the effect of the leading $l=1$ contribution.

The effect of the new contribution $\gamma_{g g}^{(1)}$ to the anomalous dimension is, on the other hand, very substantial for the gluon density: the results even fall noticeably below the NLO evolution, and also below our previous lower estimate $(D)$ at $\mathrm{NL} x_{q}$ accuracy [5]. There is no convergence so far, but the analysis of the known LO and NLO results supports some hope that the inclusion of also two further subleading terms (series), $l=2$ and $l=3$, may lead to a sufficiently stable result [13]. At this point the question arises how large a correction the presently unknown resummed $\gamma_{g q}^{(1)}$ term may introduce. Experience in NLO and the L $x$ and $\mathrm{NL} x_{q}$ resummations suggests that the impact of this contribution is of the order of $10 \%$ or less, for the evolution of both $x \Sigma$ and $x g$.

We now turn to the proton structure functions. As we are working in the DIS scheme, $x \Sigma\left(x, Q^{2}\right)$ already reflects $F_{2}$ up to the non-singlet pieces which are not relevant at small $x$ t Hence we directly turn to $F_{L}\left(x, Q^{2}\right)$. The resummed results, employing the parton densities shown in Figure 1 and the $l=1$ resummation of the coefficient functions [2],

$$
C_{L}(N)=\bar{\alpha}_{s} C_{L}^{0}(N)+\bar{\alpha}_{s}^{2} C_{L}^{1}(N)_{\mathrm{DIS}}+\sum_{k=2}^{\infty} c_{L, k} \bar{\alpha}_{s}\left(\frac{\bar{\alpha}_{s}}{N-1}\right)^{k},
$$

are depicted in Figure 2(a). Here $C_{L}^{0}$ and $C_{L}^{1}$ stand for the leading and next-to-leading order [18] coefficient functions. Note that a full NL $x$ calculation of $F_{L}\left(x, Q^{2}\right)$ requires the knowledge of the presently unknown next-order resummed coefficient function even in the DIS scheme.

The resummation corrections are exceedingly large at the lower $Q^{2}$ values shown. Here they are entirely dominated by the resummed coefficient functions. At very small values of $x$ the resummation does even violate the condition $F_{L} \leq F_{2}$ at $Q^{2} \simeq 4 \mathrm{GeV}^{2}$, thus requiring more terms in the resummation or an adjustment of the input densities at $Q_{0}^{2}$. At high $Q^{2} \gtrsim 100 \mathrm{GeV}^{2}$, due to the decrease of $\alpha_{s}$ and the parton evolution, the effects of the quarkonic anomalous dimensions and the coefficient functions are of the same order. However, besides the anomalous dimensions also the coefficient function will receive subleading corrections, which are presently unknown. To estimate the possible consequences of these terms in $C_{L}$ mentioned above, Figure 2(b) repeats the calculation illustrated by Figure 2(a), but with an estimate for those unknown contributions $C_{L} \rightarrow C_{L}(1-2[N-1])$ beyond next-to-leading order. As in the quark evolution, already a moderate correction can lead to an even drastic overcompensation of the $l=1$ effect, calling for the evaluation of the next resummation contributions to $C_{L}$.

Let us summarize: Recent results in refs. [9, [12] for the first time allow a determination of a subleading small- $x$ resummed anomalous dimension, namely the 'irreducible' part of $\gamma_{g g}$ in the unpolarized case. We have extracted the corresponding expansion coefficients in the usual DIS scheme. The coefficients of the subleading small- $x$ poles turn out to be mainly of opposite sign than the Lx pieces, and they are typically much larger in $N$-space. The numerical impact of these additional terms on the proton's parton densities and structure functions has been studied.

\footnotetext{
${ }^{1}$ The resummation of the small- $x$ terms $(\mathrm{L} x)$ for the non-singlet structure functions was performed in [17]. The corresponding corrections are smaller than $1 \%$ over the whole $x$ range.
} 
It is largest for the gluon evolution, where a substantial overcompensation of the positive leading resummation effect takes place, but less important for $x \Sigma$ and $F_{L}$, which are dominated by the quarkonic anomalous dimensions and the coefficient functions. Note, however, that the 'energydependent' contributions still need to be derived. All in all, more terms need to be calculated in the small- $x$ expansions, both of the anomalous dimensions and the coefficient functions, in order to arrive at stable resummation predictions.

Acknowledgements. We would like to thank M. Ciafaloni, L. Lipatov, S. Catani, and G. Camici for useful discussions. This work was supported in part by the German Federal Ministry for Research and Technology (BMBF) under contract No. 05 7WZ91P (0).

\section{References}

[1] Y. Balitsky and L. Lipatov, Sov. J. Nucl. Phys. 28, 822 (1978).

[2] S. Catani and F. Hautmann, Nucl. Phys. B427, 475 (1994).

[3] R.K. Ellis, F. Hautmann, and B. Webber, Phys. Lett. B348, 582 (1995).

[4] R. Ball and S. Forte, Phys. Lett. B351, 313 (1995); B358, 365 (1995);

J. Forshaw, R. Roberts, and R. Thorne, Phys. Lett. B356, 79 (1995).

[5] J. Blümlein, S. Riemersma, and A. Vogt, Nucl. Phys. B (Proc. Suppl.) 51C, 30 (1996).

[6] R. Thorne, Phys. Lett. B392, 463 (1997); hep-ph/9701241;

I. Bojak and M. Ernst, Phys. Lett. B397, 296 (1997); hep-ph/9702282.

[7] J. Blümlein and A. Vogt, hep-ph/9706371, DESY 97-106.

[8] S. Catani, M. Ciafaloni, and F. Hautmann, Nucl. Phys. B366, 135 (1991).

[9] G. Camici and M. Ciafaloni, Phys. Lett. B386, 341 (1996).

[10] V. Fadin and L. Lipatov, Yad. Fiz. 50, 1141 (1989); Nucl. Phys. B406, 259 (1993); Nucl. Phys. B477, 767 (1996);

V. Fadin, R. Fiore, and A. Quartalo, Phys. Rev. D50, 2265, 5893 (1994);

V. Fadin, R. Fiore, and M. Kotsky, Phys. Lett. B359, 181 (1995); B387, 593 (1996).

[11] V. Del Duca, Phys. Rev. D54, 989, 4474 (1996).

[12] G. Camici and M. Ciafaloni, hep-ph/9707390.

[13] J. Blümlein and A. Vogt, to appear.

[14] M. Ciafaloni, Phys. Lett. B356, 74 (1995).

[15] G. Camici and M. Ciafaloni, Nucl. Phys. B496, 305 (1997).

[16] A.D. Martin, R.G. Roberts, and W.J. Stirling, Phys. Lett. B354, 155 (1995).

[17] J. Blümlein and A. Vogt, Phys. Lett. B370, 149 (1996); Acta Phys. Pol. B27, 1309 (1996).

[18] E. Zijlstra and W. van Neerven, Nucl. Phys. B383, 525 (1992);

J. Sanchez Guillén et al., Nucl. Phys. B353, 337 (1991). 

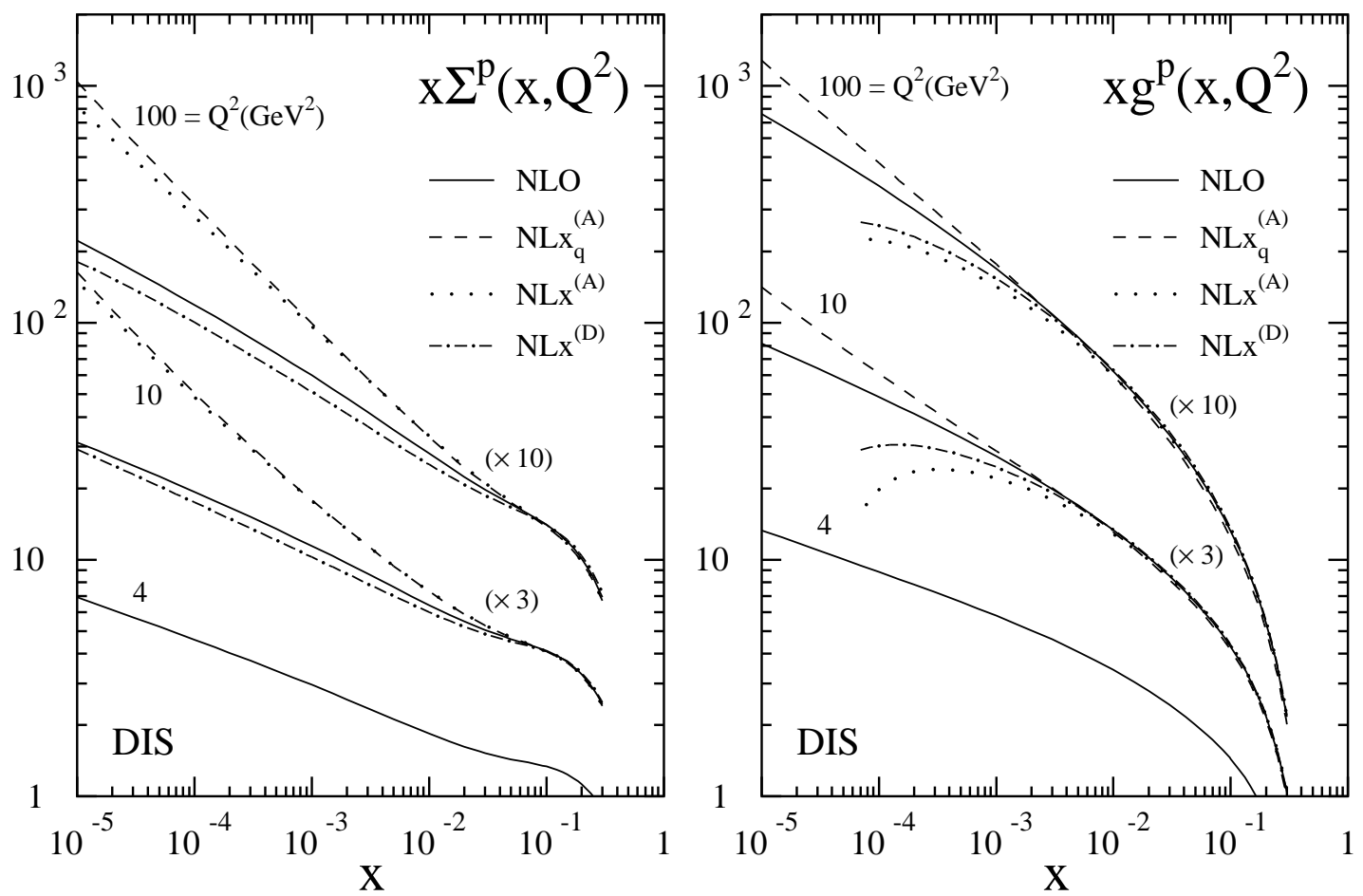

Fig. 1: The small- $x$ evolution of the singlet quark and gluon densities including the resummed $\mathrm{NL} x_{q}$ kernels [2] and the new terms of the gluon-gluon anomalous dimension [9, 12] as compared to the NLO results. Two prescriptions for implementing the momentum sum rule have been applied.
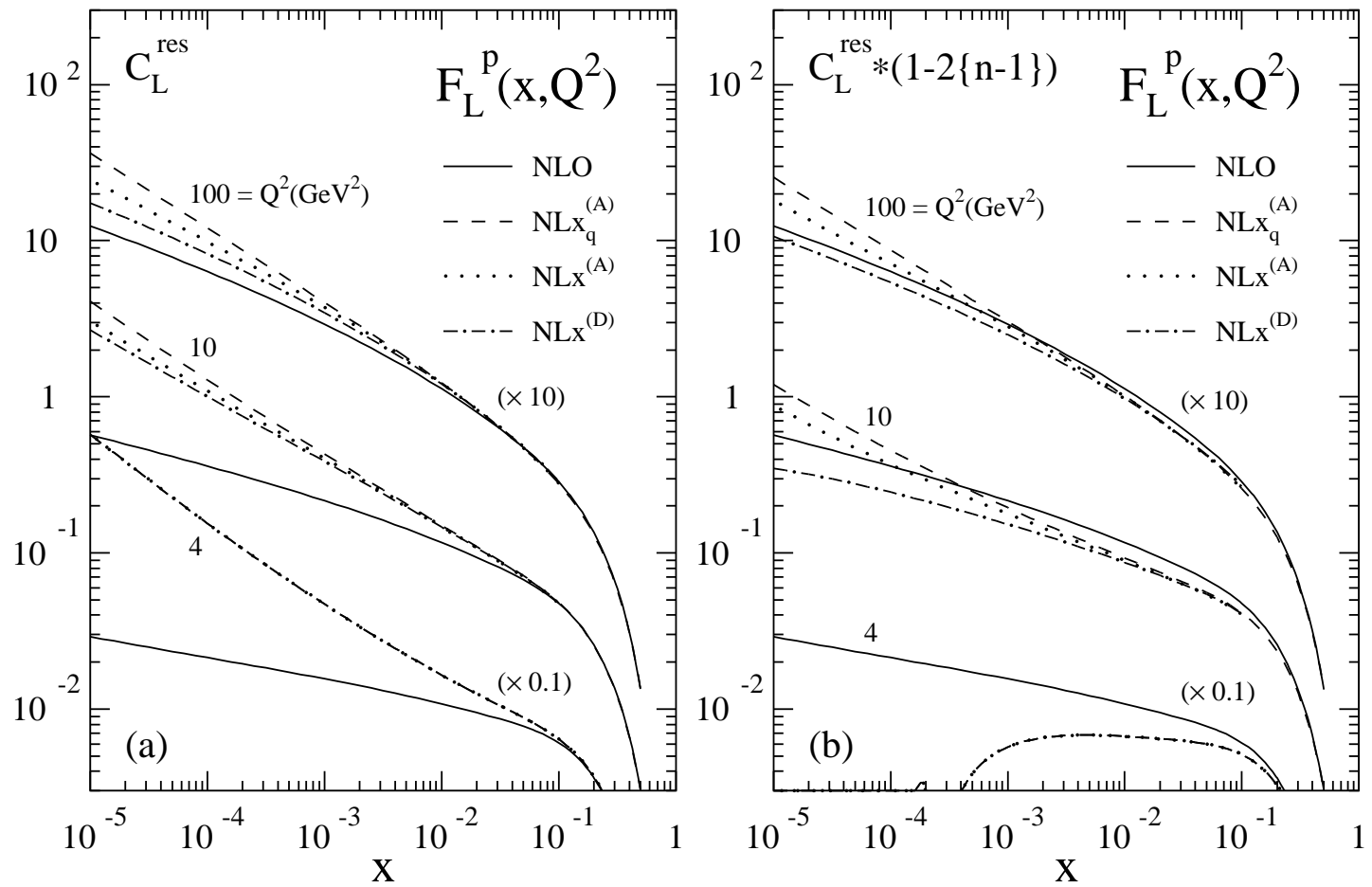

Fig. 2: The small- $x$ behavior of the longitudinal structure function for the parton distributions shown in Fig. 1. The resummed coefficient functions of ref. [2] have been employed, in (a) without, in (b) with a moderate subleading contribution. 